University of Nebraska - Lincoln

DigitalCommons@University of Nebraska - Lincoln

$8-31-2010$

\title{
Potential barrier effects in high-order harmonic generation by transition-metal ions
}

\author{
M. V. Frolov \\ Voronezh State University, Russia, frolov@phys.vsu.ru
}

N. L. Manakov

Voronezh State University, Universitetskaya pl. 1, Voronezh, 394006 Russia, manakov@phys.vsu.ru

Anthony F. Starace

University of Nebraska-Lincoln, astarace1@unl.edu

Follow this and additional works at: https://digitalcommons.unl.edu/physicsstarace

Part of the Physics Commons

Frolov, M. V.; Manakov, N. L.; and Starace, Anthony F., "Potential barrier effects in high-order harmonic generation by transition-metal ions" (2010). Anthony F. Starace Publications. 174.

https://digitalcommons.unl.edu/physicsstarace/174

This Article is brought to you for free and open access by the Research Papers in Physics and Astronomy at DigitalCommons@University of Nebraska - Lincoln. It has been accepted for inclusion in Anthony F. Starace Publications by an authorized administrator of DigitalCommons@University of Nebraska - Lincoln. 


\title{
Potential barrier effects in high-order harmonic generation by transition-metal ions
}

\author{
M. V. Frolov and N. L. Manakov \\ Department of Physics, Voronezh State University, 394006 Voronezh, Russia \\ Anthony F. Starace \\ Department of Physics and Astronomy, The University of Nebraska, Lincoln, Nebraska 68588-0299, USA
}

(Received 16 June 2010; published 31 August 2010)

\begin{abstract}
The experimental finding of significant enhancement or suppression of particular harmonics generated by the ionic component of laser-produced plasmas of transition-metal atoms is explained theoretically in terms of the standard three-step scenario for strong-field harmonic generation, taking into account the potential barrier effects that lead to a strong $3 p \rightarrow 3 d$ electric dipole transition that dominates the photoionization cross sections of the outer subshells of those ions.
\end{abstract}

DOI: 10.1103/PhysRevA.82.023424

PACS number(s): $32.80 . \mathrm{Rm}, 42.65 . \mathrm{Ky}, 32.80 . \mathrm{Wr}, 32.80 . \mathrm{Aa}$

\section{INTRODUCTION}

Over the past decade, the generation of high-order harmonics of an intense femtosecond Ti:sapphire laser by gaseous media has been widely used in various practical applications, for example, as a source of coherent extreme ultraviolet (xuv) radiation and as a tool for the production of ultrashort electromagnetic pulses for attosecond science (cf. Ref. [1] for a review). The rare gases, which have similar atomic structures, are most commonly used as the generating medium. Recent studies, however, have shown that atomic structure effects have an essential influence on the yields of high-order harmonics and their dependence on the pump laser frequency (cf., e.g., Ref. [2]). Since these atom-specific effects can lead to increased conversion efficiency $\eta$, determining and producing the optimum media for efficient high-order harmonic generation (HHG) is both a challenging and important problem. Recent results of Ganeev et al. (see the reviews in Ref. [3] and references therein) on the generation of xuv radiation by means of HHG from laser-produced plasma plumes originating from the surfaces of solid targets represents a most promising advance: since these targets can involve any atomic species that can be prepared in the solid phase, the range of possible media for HHG can be greatly extended. Several important facts were established in these plasma $\mathrm{HHG}$ experiments. First, for moderate laser intensities $\left(<10^{15} \mathrm{~W} / \mathrm{cm}^{2}\right)$, the generating medium is the ionic component of a weakly ionized plasma comprising mostly singly charged atomic ions. Second, strong, resonance-like enhancements of a single harmonic were found for a number of solid targets (with photon energies $E_{\gamma}$ up to $52.9 \mathrm{eV}$ [4]). Moreover, these enhancements arise only for those ions in which the energy $E_{\gamma}$ of the enhanced harmonic is close to the energy of an ionic transition between the ground and a quasibound (autoionizing) state having a strong oscillator strength, thereby confirming the sensitivity of the HHG yield to the atomic structure. Since the conversion efficiency for such enhanced harmonics in HHG by plasmas can achieve record values ( $\eta \approx 10^{-4}[3]$ ), a proper theoretical explanation of these enhancements would be useful for predicting the most efficient harmonics for various atomic species. Highly relevant for such a theoretical explanation is a third important experimental observation, which is that, in some cases, together with strong enhancement of the $N$ th harmonic, one observes a significant suppression of the preceding, $(N-2)$ th harmonic [4-7]. For example, along with the enhanced 29th harmonic of the Ti:sapphire laser, a considerable suppression of the 27th harmonic generated in a chromium plasma was observed in Refs. [4-6] under various focusing conditions and for various laser intensities and pulse durations.

Recently, two alternative theoretical models $[8,9]$ have been proposed to interpret such single-harmonic enhancements in HHG by laser-produced plasmas. Both models introduce a modification of the standard three-step scenario for HHG [10]. Based on the results of Ref. [11], Ref. [8] assumes the existence of a highly (and equally) populated coherent superposition of the ground and quasicontinuum (autoionizing) states. In addition to the fact that the justification for the formation of such a superposition in HHG from laser-generated plasma plumes is unclear, the enhancements in this model appear for groups of neighboring harmonics, in a way similar to that for HHG by neutral atoms in which the ground and excited bound states are in resonance [12]. [Note that autoionizing states have been shown recently to influence the phase matching of high-order harmonics, which may result in the selective enhancement of the yield of a single harmonic under some conditions [13] (see also Ref. [14]); it seems unlikely, however, that these conditions are fulfilled for the same harmonic over a range of very different experimental parameters, as has been found for the case of $\mathrm{Cr}^{+}$[4-6].] In the second model, the third (recombination) step of the three-step scenario is partitioned into two steps [9]: the capture of a laser-accelerated electron into an autoionizing state of the parent ion followed by the radiative relaxation of this state to the ground state with emission of the harmonic photon. Although this four-step model provides reasonable estimates for the ratio of the enhanced harmonic intensity to the averaged intensity of neighboring harmonics, the suppression of harmonics preceding the resonant one remains a puzzle for both of the proposed theoretical models $[8,9]$.

In this paper we show that enhancements of single harmonics with harmonic energies near the energies of autoionizing states in atoms or atomic ions, as well as the aforementioned suppression of the preceding (or, in some cases, the succeeding) harmonics, may be interpreted (at least for those harmonics in the region of the classical plateau cutoffs for 
a given laser frequency and intensity) in terms of the usual three-step scenario for the HHG process [10], without any additional assumptions or extensions.

\section{THEORETICAL BACKGROUND}

Our considerations are based on the use of the factorized formula for the HHG rates [2], which provides explicit quantum expressions for each of the three steps (i.e., ionization, propagation, and recombination) of the semiclassical three-step scenario [10]. The final (recombination) step in this formula is described by the exact (atom-specific) photorecombination cross section $\bar{\sigma}(E)$ of the laser-driven, active atomic electron (with energy $E$ ), which is captured into the ground state of the atomic target from which it was ionized (in the first step) by the strong laser field. The idea that information on the important free-atom parameter, $\bar{\sigma}(E)$, may be extracted directly from HHG measurements was proposed first in Ref. [15]. Later, the hypothesis that the HHG rate may be presented as the product of $\bar{\sigma}(E)$ and an "electron wave packet" (EWP) was confirmed numerically, based on the direct numerical solution of the time-dependent Schrödinger equation [16]. In Refs. [2,17], the explicit form for the EWP was derived analytically in the low-frequency (tunneling) limit.

As shown in Ref. [2], the analytic quantum result for the HHG rate $\mathcal{R}\left(E_{\gamma}\right)$ for a harmonic with energy $E_{\gamma}=\hbar \Omega=$ $N \hbar \omega$ located at the high-energy end of the HHG plateau has the following factorized form:

$$
\mathcal{R}\left(E_{\gamma}\right)=\mathcal{T} \mathcal{W}(E) \bar{\sigma}(E), \quad E_{\gamma}=E+\left|E_{0}\right|,
$$

where $E_{0}$ is the ground-state energy of the target atom or ion and $E$ is the active electron's energy at the moment of recombination. The factor $\mathcal{T}$ in Eq. (1) is the ionization factor describing the first (tunneling) step of the three-step scenario. It involves the tunneling rate from the initial bound state of energy $E_{0}$ in an effective static electric field. The propagation factor $\mathcal{W}$ involves an Airy function that describes interference effects between short and long electron trajectories during the course of propagation of the active electron in the laser field up to the moment of recombination. The recombination event is described in Eq. (1) by the photorecombination cross section, $\bar{\sigma}(E)$.

The factorized formula (1) was derived for a monochromatic laser pulse with fixed intensity $I$ and frequency $\omega$. For this case, the interference oscillations of the propagation factor $\mathcal{W}(E)$ lead to rapid oscillations of the HHG yield with variation of both the electron energy $E$ (or harmonic frequency $\Omega$ ) and the laser intensity $[2,17]$. Thus, in order to compare the theoretical results for HHG rates with the experimental results obtained using focused laser beams, we need to perform a focal average of the EWP, $W(E)=\mathcal{T} \mathcal{W}(E)$ [since $\bar{\sigma}(E)$ is independent of the laser parameters]. Moreover, for high laser intensities, the generating medium is rapidly depleted and these depletion effects should be taken into account in performing focal averaging. For this purpose, we employ the techniques used in Ref. [18]. In Fig. 1 we present the focal-averaged EWP $W(E)$ for $\mathrm{Cr}^{+}$ions at several peak laser intensities for a 35-fs laser pulse with wavelength $\lambda=800 \mathrm{~nm}$. As Fig. 1 shows, the focal averaging smooths out all interference effects in the EWP. This leads to similar

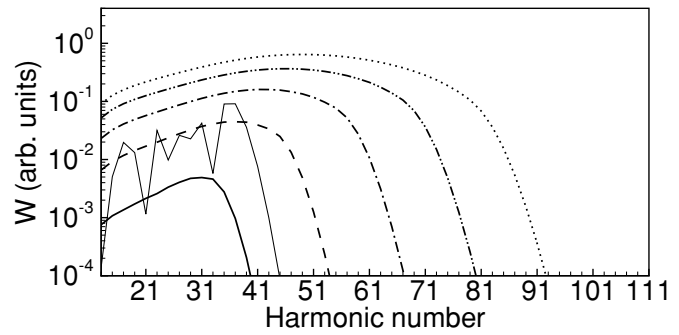

FIG. 1. The focal-averaged electron wave packet for $\mathrm{Cr}^{+}$for different intensities $I=\tilde{I} \times 10^{14} \mathrm{~W} / \mathrm{cm}^{2}$ and $\lambda=800 \mathrm{~nm}$. Solid line, $\tilde{I}=2$; dashed line, $\tilde{I}=3$; dash-dotted line, $\tilde{I}=4$; dash-dot-dotted line, $\tilde{I}=5$; dotted line, $\tilde{I}=6$. Thin solid line, electron wave packet for $\tilde{I}=2$ without focal averaging.

behaviors of the EWPs in the plateau region for different intensities, thus making our theoretical results for the harmonic yield in the plateau region only weakly sensitive to the choice of laser intensity. Therefore, any peculiarities in the HHG rates can be attributed primarily to those in the photorecombination cross sections. As is well known, for complex atoms or atomic ions these cross sections exhibit irregularities caused by Cooper minima, potential barrier and electron correlation effects, and so on [19]. In particular, for the neutral Xe atom, a significant enhancement of the energy-integrated HHG yield in the xuv region was predicted in Ref. [2]. This enhancement originates from the well-known "giant" dipole resonance in the photoionization cross section from the inner $4 d$ subshell in $\mathrm{Xe}$ that appears in the photoionization cross section for the outer $5 p$ subshell owing to interchannel coupling. As we show below, similar peculiarities, caused by Fano autoionizing resonances in the cross sections for photoionization of singly charged transition-metal ions, explain the observed enhancement or suppression of particular harmonics in the observed plasma HHG spectra.

\section{RESULTS AND DISCUSSION}

In Figs. 2(b) and 2(d) we present our calculated HHG spectra for $\mathrm{Cr}^{+}$and $\mathrm{Mn}^{+}$, respectively, and compare them with the experimental HHG results [4,7] for the case of a wavelength of $800 \mathrm{~nm}$ (Ti:sapphire laser). We are limited to presenting $\mathrm{HHG}$ spectra over only a narrow range of harmonic numbers owing to the rather scarce information available on the photoionization cross sections for atomic ions having a complicated electronic structure. The calculation of these photoionization cross sections with proper account of many-electron interactions presents a considerable technical challenge even at the present time. The photorecombination cross sections $\bar{\sigma}$ needed for the theoretical results presented in Figs. 2(b) and 2(d) were deduced using the principle of detailed balance from the corresponding photoionization cross sections $\sigma$ shown in Figs. 2(a) and 2(c). (Concerning the use of this principle for the case of Fano resonances in photoionization cross sections, see Ref. [20].) In Fig. 2 we have plotted the photoionization cross sections for $\mathrm{Cr}^{+}$and $\mathrm{Mn}^{+}$as functions of photon energy $E_{\gamma}$ on the same energy scale as the corresponding HHG spectra so that in each case the correspondence between the energy-dependent structure in 

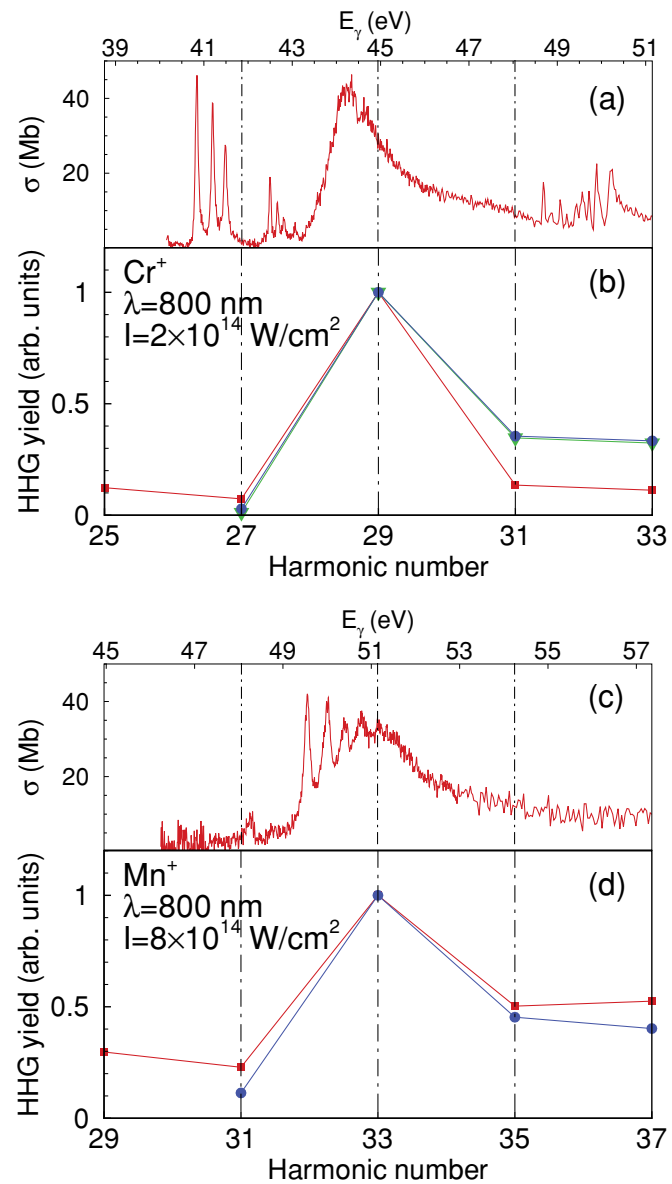

FIG. 2. (Color online) Comparisons of photoionization cross sections $\sigma$ and HHG spectra (for $\lambda=800 \mathrm{~nm}$ and intensities indicated in the panels) of (a,b) $\mathrm{Cr}^{+}$and (c, d) $\mathrm{Mn}^{+}$. The experimental results for $\sigma$ in panels (a) and (c) are taken from the database in Ref. [21]. Symbols for the HHG spectra presented in panels (b) and (d) are as follows: Squares (red), results from Ganeev et al. (cf. Fig. 4 in Ref. [4] for $\mathrm{Cr}^{+}$and Fig. 3 in Ref. [7] for $\mathrm{Mn}^{+}$); circles (blue), present results for $\beta=0$; triangles (green), present results for $\mathrm{Cr}^{+}$ with the asymmetry parameter $\beta$ taken from Ref. [22].

the photoionization cross section and that in the HHG spectrum is obvious.

Note that $\bar{\sigma}(E)$ in Eq. (1) is the differential photorecombination cross section for an electron with momentum $\mathbf{p}(p=\sqrt{2 m E})$, directed along the polarization axis of the laser field $\mathbf{F}(t)$, that recombines to the ground state with emission of a harmonic photon (of energy $E_{\gamma}$ ), whose polarization is the same as that of the field $\mathbf{F}(t)[2,17]$. Thus, to obtain the photorecombination cross section $\bar{\sigma}(E)$ we need both the total photoionization cross section $\sigma$ and the angular distribution asymmetry parameter $\beta(E)$. The total photoionization cross section is available from both experimental measurements [21,23-25] and theoretical calculations [22,26-28], whereas the asymmetry parameters are mostly available from theoretical calculations [22,27,29]. However, our results for $\mathrm{Cr}^{+}$in Figs. 2(b) and 3(a) (using the theoretical values for $\beta$ from Ref. [22] and experimental data for $\sigma$ from the database [21]) show that neglect of the asymmetry parameter does not change the calculated HHG
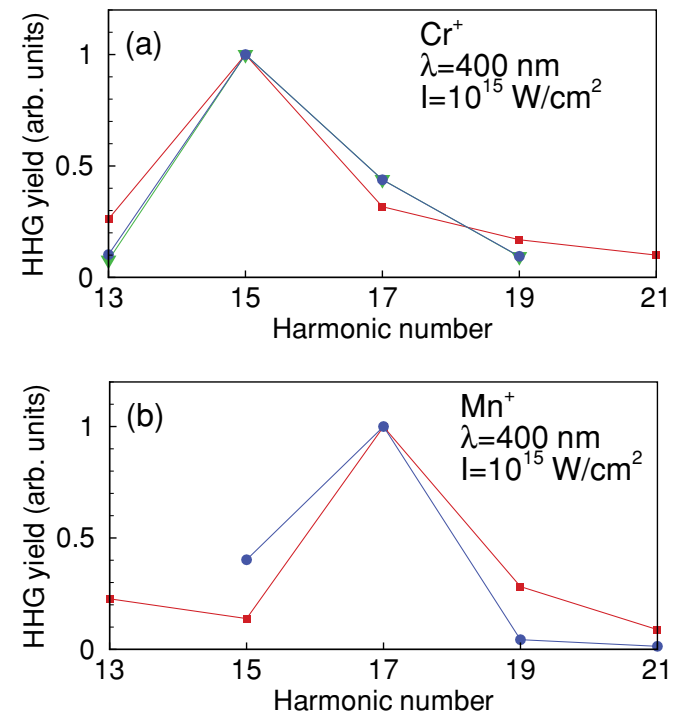

FIG. 3. (Color online) Same as Figs. 2(b) and 2(d), but for $\lambda=$ $400 \mathrm{~nm}$ and $I=10^{15} \mathrm{~W} / \mathrm{cm}^{2}$. For Ganeev et al. results, compare Fig. 6 in Ref. [4] for $\mathrm{Cr}^{+}$and Fig. 2 in Ref. [4] for $\mathrm{Mn}^{+}$.

spectrum significantly in the resonance region, so we have neglected $\beta(E)$ in our lowest-order approximation. Also, we used the experimental data [21] for the total photoionization cross section of $\mathrm{Mn}^{+}$, including ionization of both $4 s$ and $3 d$ electrons.

Considerable enhancements of particular, single harmonics were observed in $\mathrm{HHG}$ from plasma plumes generated by a 400-nm laser from $\mathrm{Cr}$ and $\mathrm{Mn}$ targets [4,30]. In Fig. 3 we compare the experimental results with our theoretical predictions for both the resonantly enhanced harmonic and its neighboring harmonics using experimental data [21] for the total photoionization cross sections. Since the widths $\Gamma$ of Fano profiles are $1.2 \mathrm{eV}$ (for $\mathrm{Cr}^{+}$) and $1.8 \mathrm{eV}$ (for $\mathrm{Mn}^{+}$) [24], while the distance between two neighboring harmonics for $\lambda=400 \mathrm{~nm}$ is $3.1 \mathrm{eV}$, the harmonics neighboring the resonant one (i.e., the 15th harmonic for $\mathrm{Cr}^{+}$and the 17th harmonic for $\mathrm{Mn}^{+}$) are, in fact, outside of the Fano profile region. However, the resonant enhancement of the central harmonics is clearly visible in both panels in Fig. 3. Moreover, theoretical and experimental results for the ratio of the intensity of the enhanced harmonic to the average of its two nearest neighbors are in reasonable agreement.

The asymmetric resonance features in the photoionization cross sections for $\mathrm{Cr}^{+}$and $\mathrm{Mn}^{+}$that underlie our interpretation of the experimental laser-plasma HHG spectra of Ganeev et al. $[4,7]$ stem from the by now well-known potential barrier effects that have been found in the photoionization spectra of not only the transition-metal atoms but also the lanthanide atoms having respectively unfilled $d$ and $f$ subshells [31-33] (see also the reviews in Refs. [19,34] and references therein). Consider specifically the case of $\mathrm{Cr}^{+}$, whose ground-state configuration is $3 p^{6} 3 d^{5}\left({ }^{6} S\right)$ in $L S$ coupling. The outer $3 d$ subshell electrons, which are the ones that are driven by the laser to generate harmonics, have a photoionization cross section that is dominated by the one-electron electric dipole transition from the $3 p$ subshell to the $3 d$ subshell, that is, by the transition $\mathrm{Cr}^{+} 3 p^{6} 3 d^{5}\left({ }^{6} S\right)+\gamma \rightarrow \mathrm{Cr}^{+} 3 p^{5}\left({ }^{2} P\right) 3 d^{6}\left({ }^{5} D\right)\left({ }^{6} P\right)$. 
This transition is very strong for two reasons: First, there are six $3 p$ subshell electrons and five $3 d$ subshell vacancies, making the transition statistically strong [32]. Second, owing to the large effective potential barrier for $d$ electrons, only the occupied $3 d$ subshell orbital wave function is located inside the effective potential barrier, ensuring strong wave function overlap with the $3 p$ subshell orbital wave function, while higher-energy $d$ orbitals are kept out of the radial region occupied by the $3 p$ subshell owing to the large centrifugal barrier component of the effective radial potential [31]. In the energy region of interest here, the strongly populated intermediate state then autoionizes to the continuum states resulting from photoionization of the outer $3 d$ subshell, as follows [33]: $\mathrm{Cr}^{+} 3 p^{5}\left({ }^{2} P\right) 3 d^{6}\left({ }^{6} P\right) \rightarrow \mathrm{Cr}^{2+} 3 p^{6} 3 d^{4}\left({ }^{5} D\right)+\epsilon \ell$, where the dominant final state channel is that for $\ell=3$.

In general, the parametrization of the $3 d$ subshell cross section in the region of the $3 p \rightarrow 3 d$ resonance is quite complicated, owing on the one hand to the difficulty of properly characterizing the many interacting resonance states that autoionize to many continuum channels $[32,33]$ and on the other hand to the fact that parametrization of partial cross sections requires an additional set of parameters $[35,36]$ beyond those necessary to describe the total cross section [37,38]. For $\mathrm{Cr}^{+}$in the case of $L S$ coupling, however, one has a single resonance state interacting primarily with a single continuum channel, in which case the parametrization of the $3 d$ subshell partial cross section in the resonance region reduces to the usual Fano form:

$$
\sigma(E)=\sigma_{0}(q+\epsilon)^{2} /\left(1+\epsilon^{2}\right),
$$

where $\sigma_{0}$ is the cross section outside the resonance region, $q$ is the Fano profile parameter [37], and $\epsilon$ is a reduced energy [37],

$$
\epsilon=\left(E-E_{r}\right) /(\Gamma / 2)
$$

where $E_{r}$ is the resonance energy and $\Gamma$ is its full width at half maximum. A sum of such resonance profiles (in the case of many intermediate state resonances) has been found to describe well the photoionization cross sections of a number of transition-metal atoms and ions (see, e.g., Ref. [34]). One sees clearly that this profile is asymmetric with respect to the resonance energy. This asymmetry results in a similar asymmetry in the photorecombination cross section that determines the HHG spectrum.

The case of $\mathrm{Mn}^{+}$is slightly more complicated to analyze since its ground state has the configuration $3 p^{6} 3 d^{5}\left({ }^{6} S\right) 4 s\left({ }^{7} S\right)$ in $L S$ coupling. The presence of the $4 s$ electron leads to additional structure in the photoionization cross sections of the $3 d$ and $4 s$ subshells [39], with the $3 d$ subshell partial cross section being by far the dominant one in the vicinity of the $3 p \rightarrow 3 d$ resonance transition. However, the broad structure of the photoionization cross section is very similar to that for $\mathrm{Cr}^{+}$, so our predictions for the asymmetric resonance features of the HHG spectra shown in Figs. 2 and 3 are also similar.

\section{CONCLUSIONS}

In conclusion, we have successfully reproduced the main features observed in experiments on HHG from plasmas produced by the laser ablation of solid $\mathrm{Cr}$ and $\mathrm{Mn}$ targets. For both 800 - and 400-nm wavelengths, these features are caused by atomic structure effects in the radiative recombination cross sections of $\mathrm{Cr}^{2+}$ and $\mathrm{Mn}^{2+}$ ions (or, equivalently, in the photoionization cross sections of $\mathrm{Cr}^{+}$and $\mathrm{Mn}^{+}$ions). These effects are predicted by the factorization formula (1) for HHG rates. The experimental measurements of these rates serve to complement the measurements of Cooper minima in $\mathrm{HHG}$ from neutral atoms [40]. In contrast to the Cooper minima in HHG rates, however, which have a predominantly singleelectron origin, the plasma HHG results can be regarded as the first experimental evidence of genuine multielectron atomic dynamics in HHG spectra. Since this dynamics in principle cannot be described by the commonly used single-activeelectron approximation to the time-dependent Schrödinger equation for HHG processes, the search for atomic targets permitting enhanced harmonic generation in the xuv domain should stimulate accurate multielectron calculations of photoionization cross sections from outer (sub)shells of complex atoms and ions in order to provide an accurate description of the strongly resonant transitions that underlie the enhanced yield of particular high-order harmonics.

\section{ACKNOWLEDGMENTS}

We gratefully acknowledge fruitful discussions with V. K. Dolmatov on the photoionization of $\mathrm{Cr}^{+}$and $\mathrm{Mn}^{+}$ions and with R. A. Ganeev on the experimental aspects of HHG from laser-generated plasmas. This work was supported in part by the Russian Foundation for Basic Research under Grant No. 10-02-00235, by Grant No. MK-2425.2009.2 (M.V.F) of the President of the Russian Federation, and by NSF Grant No. PHY-0901673.
[1] F. Krausz and M. Ivanov, Rev. Mod. Phys. 81, 163 (2009).

[2] M. V. Frolov, N. L. Manakov, T. S. Sarantseva, M. Yu. Emelin, M. Yu. Ryabikin, and A. F. Starace, Phys. Rev. Lett. 102, 243901 (2009).

[3] R. A. Ganeev, J. Phys. B 40, R213 (1997); Usp. Fiz. Nauk 179, 65 (2009) [Sov. Phys. Usp. 52, 55 (2009)].

[4] R. A. Ganeev, L. B. Elouga Bom, J.-C. Kieffer, and T. Ozaki, Phys. Rev. A 75, 063806 (2007).

[5] R. A. Ganeev, M. Suzuki, M. Baba, and H. Kuroda, Appl. Phys. Lett. 86, 131116 (2005).
[6] R. A. Ganeev, P. A. Naik, H. Singhal, J. A. Chakera, and P. D. Gupta, Opt. Lett. 32, 65 (2007).

[7] R. A. Ganeev, M. Suzuki, M. Baba, and H. Kuroda, Phys. Rev. A 76, 023805 (2007).

[8] R. A. Ganeev and D. B. Milošević, J. Opt. Soc. Am. B 25, 1127 (2008); D. B. Milošević, Phys. Rev. A 81, 023802 (2010).

[9] V. Strelkov, Phys. Rev. Lett. 104, 123901 (2010).

[10] K. J. Schafer, B. Yang, L. F. DiMauro, and K. C. Kulander, Phys. Rev. Lett. 70, 1599 (1993); P. B. Corkum, ibid. 71, 1994 (1993).

[11] D. B. Milošević, J. Opt. Soc. Am. B 23, 308 (2006); J. Phys. B 40, 3367 (2007). 
[12] R. Taïeb, V. Véniard, J. Wassaf, and A. Maquet, Phys. Rev. A 68, 033403 (2003); I. A. Ivanov and A. S. Kheifets, ibid. 78, 053406 (2008).

[13] I. A. Kulagin and T. Usmanov, Opt. Lett. 34, 2616 (2009).

[14] L. B. Elouga Bom, F. Bouzid, F. Vidal, J.-C. Kieffer, and T. Ozaki, J. Phys. B 41, 215401 (2008).

[15] J. Itatani, J. Levesque, D. Zeidler, H. Niikura, H. Pepin, J. C. Kieffer, P. B. Corkum, and D. M. Villeneuve, Nature (London) 432, 867 (2004).

[16] T. Morishita, A. T. Le, Z. Chen, and C. D. Lin, Phys. Rev. Lett. 100, 013903 (2008); A. T. Le, T. Morishita, and C. D. Lin, Phys. Rev. A 78, 023814 (2008); C. D. Lin, A. T. Le, Z. Chen, T. Morishita, and R. Lucchese, J. Phys. B 43, 122001 (2010).

[17] M. V. Frolov, N. L. Manakov, T. S. Sarantseva, and A. F. Starace, J. Phys. B 42, 035601 (2009).

[18] R. Reichle, H. Helm, and I. Yu. Kiyan, Phys. Rev. A 68, 063404 (2003); B. Bergues, Z. Ansari, D. Hanstorp, and I. Yu. Kiyan, ibid. 75, 063415 (2007).

[19] A. F. Starace, in Handbuch der Physik, edited by S. Flügge and W. Mehlhorn (Springer-Verlag, Berlin, 1982), Vol. XXXI, p. 1; M. Ya. Amusia, Atomic Photoeffect (Plenum, New York, 1990).

[20] D. Nikolić, T. W. Gorczyca, and N. R. Badnell, Phys. Rev. A 79, 012703 (2009); 81, 030501(R) (2010).

[21] H. Kjeldsen (unpublished), [http://www.phys.au.dk/ hkj/data. $\mathrm{html}$.

[22] V. K. Dolmatov and S. T. Manson, J. Phys. B 30, L517 (1997).

[23] J. B. West, J. E. Hansen, B. Kristensen, F. Folkmann, and H. Kjeldsen, J. Phys. B 36, L327 (2003).

[24] H. Kjeldsen, F. Folkmann, B. Kristensen, J. B. West, and J. E. Hansen, J. Phys. B 37, 1321 (2004).
[25] J. E. Hansen, H. Kjeldsen, F. Folkmann, M. Martins, and J. B. West, J. Phys. B 40, 293 (2007).

[26] V. K. Dolmatov, J. Phys. B 25, L629 (1992).

[27] V. K. Dolmatov and M. Ya. Amusia, J. Phys. B 27, L281 (1994).

[28] V. K. Dolmatov, E. Guler, and S. T. Manson, Phys. Rev. A 76, 032704 (2007).

[29] V. K. Dolmatov and S. T. Manson, Phys. Rev. A 58, R2635 (1998).

[30] R. A. Ganeev, L. B. Elouga Bom, and T. Ozaki, Appl. Phys. Lett. 91, 131104 (2007).

[31] J. L. Dehmer, A. F. Starace, U. Fano, J. Sugar, and J. W. Cooper, Phys. Rev. Lett. 26, 1521 (1971).

[32] A. F. Starace, Phys. Rev. B 5, 1773 (1972).

[33] L. C. Davis and L. A. Feldkamp, Solid State Commun. 19, 413 (1976).

[34] B. Sonntag and P. Zimmermann, Rep. Prog. Phys. 55, 911 (1992).

[35] A. F. Starace, Phys. Rev. A 16, 231 (1977).

[36] C.-N. Liu and A. F. Starace, Phys. Rev. A 59, 1731(R) (1999); Phys. Essays 13, 215 (2000).

[37] U. Fano, Phys. Rev. 124, 1866 (1961).

[38] U. Fano and J. W. Cooper, Rev. Mod. Phys. 40, 441 (1968).

[39] V. K. Dolmatov, J. Phys. B 26, L79 (1993).

[40] S. Minemoto, T. Umegaki, Y. Oguchi, T. Morishita, A. T. Le, S. Watanabe, and H. Sakai, Phys. Rev. A 78, 061402(R) (2008); H. J. Wörner, H. Niikura, J. B. Bertrand, P. B. Corkum, and D. M. Villeneuve, Phys. Rev. Lett. 102, 103901 (2009). 